\title{
Adverse effects of using dolutegravir HIV in pregnant women and their newborn
}

\begin{abstract}
Introduction: The adverse effects of dolutegravir on the mother and the fetus not yet have been widely studied; the integrate inhibitor antiretroviral drug was to prevent vertical transmission of HIV.
\end{abstract}

Objective: To identify the adverse effects of the use of dolutegravir That Have occurred in HIV-positive pregnant women and their children.

Methodology: A scoping review we performed in the Medline, Cochrane Library and EBSCO databases, with the MeSH terms dolutegravir and pregnancy.

Eligibility criteria: Clinical trials and observational studies conducted in pregnant women with HIV Were Treated with dolutegravir included. The search was limited to articles in Inglés and Spanish and published Until September 12018.

Results: The bibliographic search obtained seven investigations. Two studies report mild adverse effects in the mother. Six studies related to pregnancy outcomes described and delivery, with preterm delivery and small for age stational being the MOST reported. Four cases of neural tube defects in four studies we reported.

Discussion: There is not enough scientific evidence to associate the use of dolutegravir with the appearance of adverse effects on the mother and her children.

Keywords: dolutegravir, pregnancy, adverse effects, newborn
Volume 10 Issue 5 - 2019

Lorena Del Carmen Paredes J,' Isabel Cristina Vargas S,' Diana Patricia Cabrera Ch,' Eduardo Mahecha R,' Jorge Andrés Ramos $C^{1,2}$

'Department of Epidemiology, University Surcolombiana, Colombia

${ }^{2}$ Graduate School Doctorate in Public Health, University CES, Colombia

Correspondence: Eduardo Mahecha R, Department of Epidemiology, University Surcolombiana, Neiva, Huila, Colombia, Emailedmahecha97@gmail.com

Received: September 22, 2019 | Published: October II, 2019
Abbreviations: APRI, antiretroviral pregnancy registry International; EMA, European medicines agency; FDA, food and drug administration; CEEC, patient exposure, comparison and results; $\mathrm{MeSH}$, medical subject headings; ALT, alanine aminotransferase; AST, aspartate aminotransferase; ART, antiretroviral therapy

\section{Introduction}

The dolutegravir is a drug belonging to the family of integrase inhibitors, used in the treatment of disease by HIV HIV. ${ }^{1}$ has been used by a large proportion of patients with HIV that has been administered to pregnant women in order to prevent vertical transmission. ${ }^{2}$

Being part of a relatively new drug family, performing blunt studies detailing the pharmacokinetics of this drug in pregnancy ${ }^{2}$ and describing the adverse effects presented in the mother and children born to HIV positive mothers are not evidence, for therefore this research aims to contribute to reducing the knowledge gap about the use of that drug.

HIV infection itself does not increase the risk of congenital malformations. ${ }^{3,4}$ The risk of congenital malformations of antiretrovirals has been described in observational studies and a collaborative record of women exposed to antiretroviral therapy, the Antiretroviral Pregnancy Registry International (APRI), with data from over 5,000 patients exposed to ART between 1989 and 2007 is a $2.6 \%$ prevalence of birth defects per 100 live births among patients receiving ART at any time during pregnancy., 4

Major health agencies worldwide recognition as the WHO, the
European Medicines Agency (EMA) and the Federal Agency for Food and Drug Administration (FDA) issued statements in May this year on precaution of using dolutegravir ${ }^{6}$ in pregnant women with HIV, because the results of a study that found 4 cases of children born with neural tube defects such as spina bifida in children of mothers who took dolutegravir during pregnancy. ${ }^{7}$

Because of the impact that could cause the use of this medicine in breast population with possible consequences for their children, it is important to do this review to identify adverse effects that could occur in both the expectant mother and the newborn, in order to establish precautions in the use of dolutegravir.

Therefore a review of the literature arises with the aim of identifying the adverse effects that have occurred in both HIV-positive pregnant women and their children, associated with use of dolutegravir.

\section{Methodology}

Search strategy guide was followed to conduct a scoping review in order to ensure a standard and quality in the presentation of the results of the review.

A search was conducted in the scientific literature following the scheme of Patient Exposure, Comparison and Results (CEEC). Participants were pregnant women with HIV and their children. The exhibition was the use of dolutegravir. The primary outcome was the occurrence of adverse events associated with administration of dolutegravir during pregnancy. The main objective was to determine the occurrence of adverse events in pregnant women with HIV, such 
as depression, mental disorder, gastrointestinal effects, among others; and their children, as the presence of congenital malformations.

The search was conducted in the databases Medline, EBSCO and Cochrane library, using the terms of the Medical Subject Headings $(\mathrm{MeSH})$ dolutegravir and pregnancy. The search was limited to humans, no restrictions on sample size were presented; there were restrictions on language to English and Spanish, and included studies published until 1 September 2018.

\section{Inclusion criteria}

Clinical trials, observational studies and summaries of work that included pregnant women with HIV who led dolutegravir treatment, and the children of HIV-infected women during pregnancy took dolutegravir were included.

As a source of information for gray literature, it took into account: congresses, symposia, thematic experts, abstracts and list of references.

Two researchers reviewed the titles and abstracts of retrieved studies to assess eligibility criteria. After reviewing the titles and abstracts, articles were reviewed in full text to determine eligibility criteria. Differences in Articles were resolved by discussion and consensus. If they persisted differences were a result of a third researcher.

\section{Study selection}

Search databases yielded 72 records; after removing duplicates, 69 were selected to review the title and summary. Of these, 62 were excluded and reviewed in full text 4 . In the search for references and products presented at scientific events additional 3 studies were included. ${ }^{7}$ work finally met the eligibility criteria for the review (Figure 1).

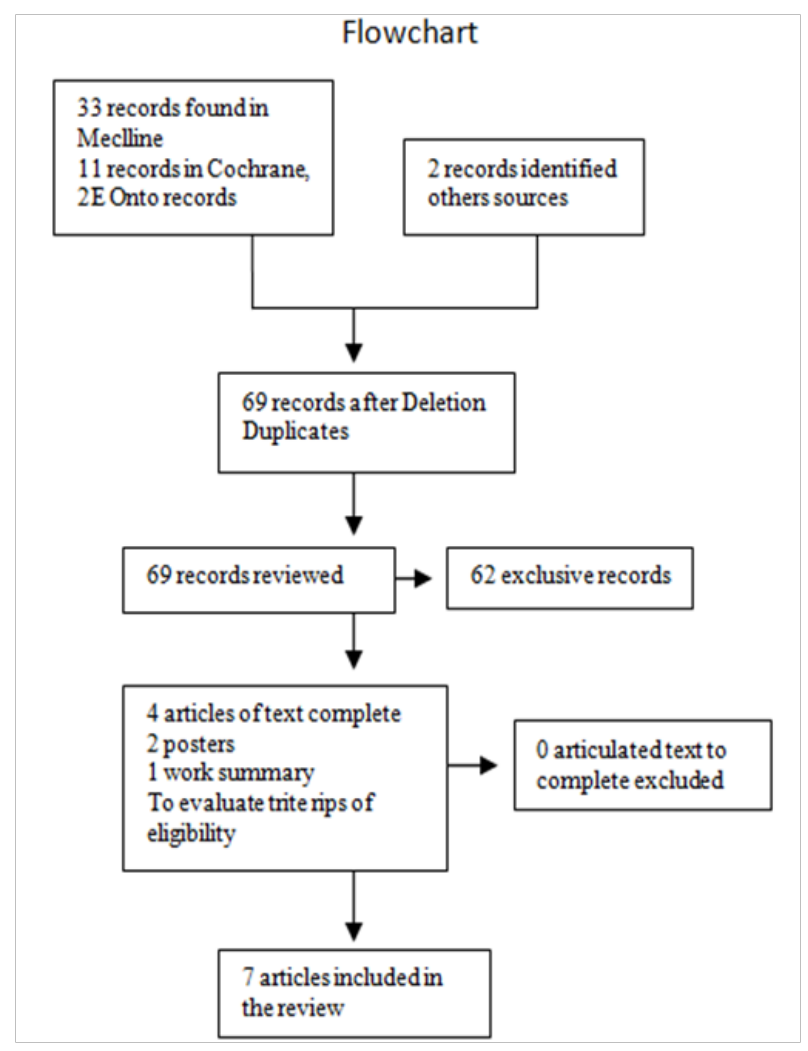

Figure I Flow chart.
The most frequent reasons for rejecting the investigations were:

a. Pregnant women without condition

b. Patients with no treatment dolutegravir

c. 2 posters included any of the scarce information about the population extracted with cases according to the outcome in the newborn and the mother and related pregnancy and childbirth.

\section{Data extraction}

The item information was extracted by three reviewers, using an established format containing the following variables: lead author, year of publication, geographic location, population, study design, dose dolutegravir, outcomes in pregnant women, pregnancy-related and delivery, and outcomes in the newborn.

\section{Synthesis of information}

Qualitative analysis of each of the articles was performed, considering the characteristics of the studies, the population level of exposure and outcomes or adverse events experienced.

\section{Results}

The literature search yielded 7 Research. The studies were published between 2016- 2018, and were carried out in Botswana, ${ }^{8}$ United States, ${ }^{9} 10$ Sweden, ${ }^{11}$ England, ${ }^{12}$ and a cohort of European

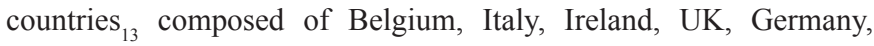
Holland, Switzerland and Spain. $100 \%$ of the studies were published in English.

Of the studies found, the adverse effects were classified according to the outcome in the mother outcome in the newborn and outcomes related to pregnancy and childbirth (Table 1).

\section{Denouement in the mother}

The study reported Borhnede 6 cases (16\%) with moderate increase of liver enzymes such as alanine aminotransferase (ALT) and aspartate aminotransferase (AST) and a case of pre-eclampsia $(2.7 \%)$. eleven

US Mulligan reported 3 maternal (14.2\%) who had anemia, 2 patients with preeclampsia $(9.5 \%)$, a headache patient $(4.7 \%)$, a case with proteinuria $(4.7 \%)$ and a mother who presented blurred vision $(4.7 \%){ }^{14}$

Of the 7 studies found none reported depression and mental disorders in the mother (Table 1).

\section{Outcomes in the newborn}

Congenital malformations in 4 studies reported:

Zash Bostwana reported in 4 patients $(0.9 \%)$ of neural tube defects in 426 live births. ${ }^{15}$

Thorne reported study of 81 children studied four congenital malformations: a case of congenital heart malformation (1.2\%), one with a history of hexadactilia parent equal status $(1.2 \%)$ a case dorsal case hyperpigmentation $(1.2 \%)$ a case ankyloglossia $(1.2 \%) .{ }^{13}$

Grayhack in the United States reported 2 cases; a child with fetal hydrops (1.7\%) and a case of cardiac malformation (1.7\%)(17). And in the United States, Mulligan 10 children studied reported 4 cases with congenital malformations; a case of pulmonary vein abnormalities $(10 \%)$, a case of kidney abnormalities, polycystic kidney disease, and cystic fibrosis $(10 \%)$, a case of tremor congenital chin $(10 \%)$ and a case of terminal fibrolipoma phylum $(10 \%)^{19}$ (Table 1$)$. 
Table I Characteristics of included studies

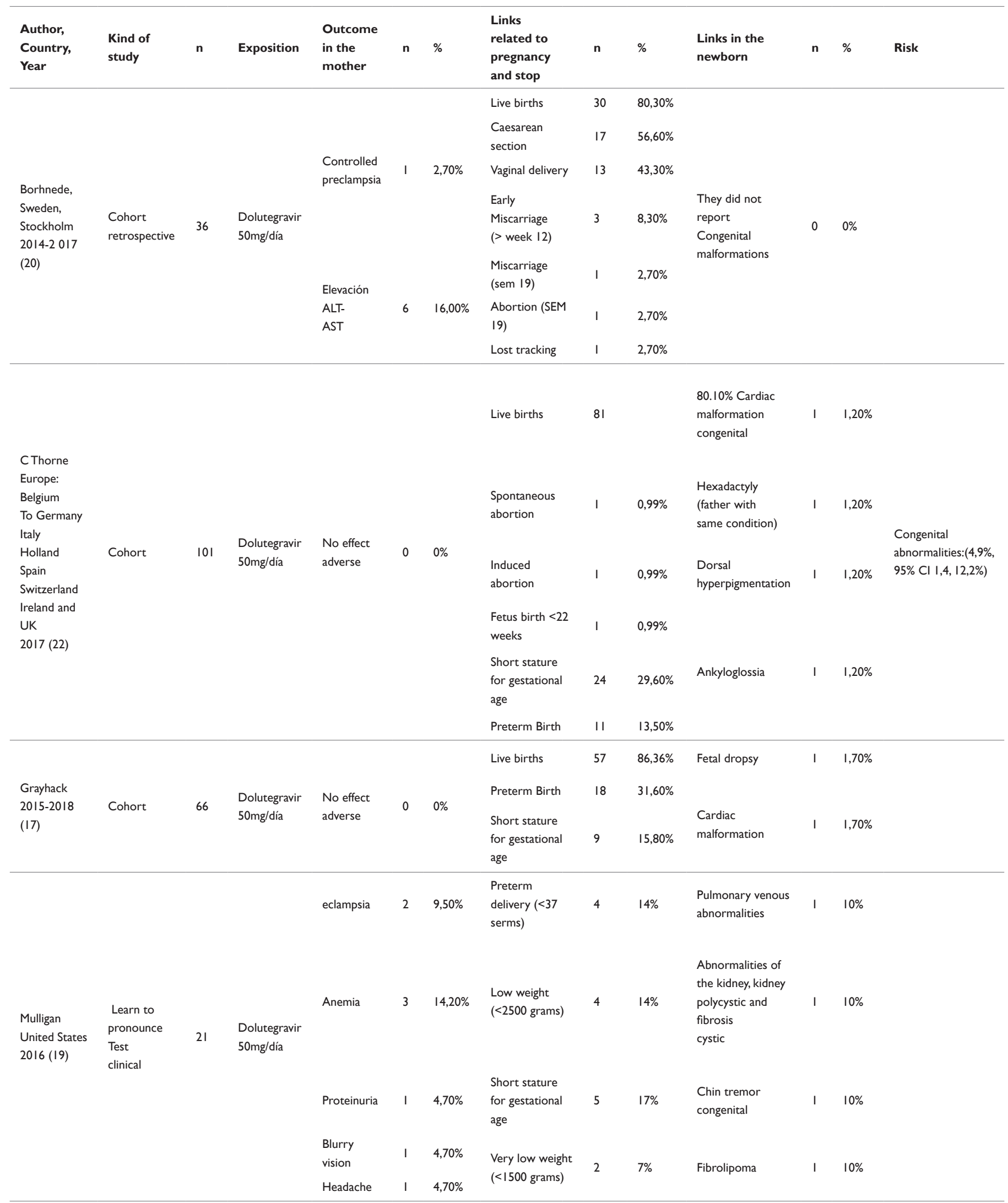


Table Continues...

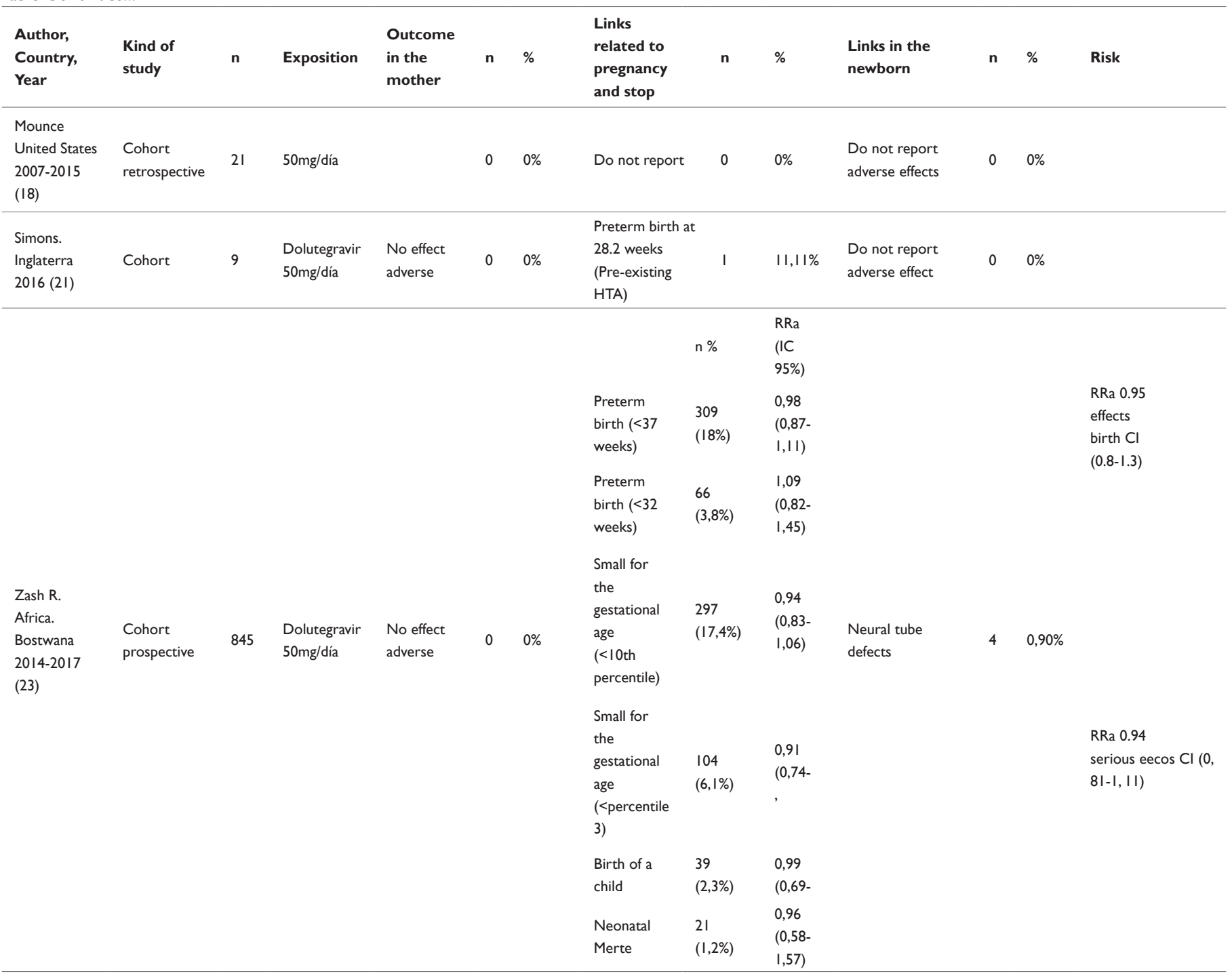

\section{Outcomes related to pregnancy and childbirth}

6 studies describing outcomes related to pregnancy and childbirth, where most events reported were preterm birth and small for gestational age were found. In 3 studies $^{15-17}$ the highest number of cases of preterm birth and small for gestational age was observed.

Zash described in Bostwana 401 cases small for gestational age of which 297 (17.4\%) were in the 10th percentile RRa (0.94) 95\% (0.83 to 1.06$)$, and 104 (6.1\%), in the 3rd percentile RRa (0.91) 95\% (0.74-1.13). In addition 375 parts of pre terms which 309 (18\%) were before 37 weeks RRa (0.98) 95\% (0.87 to 1.11$)$ and 66 (3.8\%) before 32 weeks RRa (09/01) 95\% (0.82-1.45) as not being statistically significant dolutegravir not represent risk factor for an adverse event or a serious outcome. ${ }^{7}$

Thorne study reported in $24(29.6 \%)$ cases of low height for gestational age and 11 parts pre terms (13.5\%). In 13 of 18 cases Grayhack $(31.6 \%)$ of preterm labor and 9 cases $(15.8 \%)$ of low height for gestational age were presented.

Only 2 studies report cases of spontaneous abortions and volunteers. Stockholm (Sweden) A retrospective study was conducted during the years 2014-2017 that included 36 pregnant women, where abortions were 3 spontaneous preterm $(8 \%)$ and a miscarriage. ${ }^{11}$ Only one study, Mounce reported no adverse effects on the mother or the newborn. ${ }^{9}$

\section{Discussion}

There is not enough scientific evidence to demonstrate the severe adverse reactions dolutegravir use in pregnant women with HIV. Only 7 studies were found and these adverse events were assessed different, so the results are very different in each study.

One study reported six cases of elevated liver enzymes 11 and another study reported a case of headache ${ }^{14}$ corresponding with that described in the literature on the adverse effects of dolutegravir; 2 studios to be alone with these results more epidemiological studies to assess the adverse effects of dolutegravir on the mother and newborn are needed.

Stunting for gestational age is a result of poor maternal environment, intrinsic fetal abnormalities, congenital infections or other forms of fetal malnutrition ${ }^{14}$; Preterm birth is a complex disorder with factors 
of various risk include a history of preterm birth before, uterine abnormalities, multiple gestation, maternal medical complications, low rate of pre-pregnancy body mass, gestational bleeding, low socioeconomic status, minority racial origin, behavioral habits such as smoking, alcohol, drug abuse, stressful events, limited or no prenatal care, periodontal disease and infections. ${ }^{15,16,18}$ This makes it difficult to associate small for gestational age with the use of dolutegravir.

Adverse effects to antiretroviral therapy (ART) are common, some may be manageable and others can be serious. 18 According to a Swiss study, there is a high incidence of toxic effects associated with the administration of antiretroviral drugs; In this study, 1160 patients $47 \%$ of subjects showed clinical symptoms such as fatigue and headache; Gastrointestinal effects such as nausea diarrhea and vomiting, nephrolithiasis associated with indinavir, lipodystrophy associated with stavudine, disturbances of mood and sleep-related lamivudine and efavirenz, and $27 \%$ adverse effects observed in laboratory tests as increased levels lipid, bilirubin, alkaline phosphatase and related anemia zidovudine. ${ }^{14}$

Among the adverse effects associated with the use of dolutegravir they have been reported impaired liver function tests, sleep disturbances, headache and nausea, neuropsychiatric symptoms such as depression. ${ }^{19-22}$

\section{Methodological aspects of the included studies}

The number of studies have been performed is reduced. Furthermore, the sample size of the included studies was varied from 2 to 845 pregnant patients, so it is considered insufficient to show the correlation between adverse effects such as congenital malformations and using dolutegravir during pregnancy.

\section{Limitations of the review}

The main limitation of this review is that it does not provide sufficient evidence to answer the research question. In addition the limited published information 2 poster so I can not access the document was obtained in full text were included.

\section{Advantages of the review}

The main advantage of the study is a comprehensive literature search was conducted to find all published studies. Furthermore, not only the outcome was evaluated in the mother but also in the newborn. It is also considered that due to the absence of literature, this is the first review of this topic, which would be one of the great advantages of this review. Using a systematic review because using it can collect and analyze the evidence that is available to all.

\section{Implications for research}

It is important that more intervention studies are conducted with better inclusion and exclusion criteria to have scientific evidence and to relate the association between the use of dolutegravir during pregnancy and adverse effects on the mother and child.

\section{Implications for medical practice}

According to these results could not implement protocols or management guidelines where indicated whether it is appropriate to initiate antiretroviral therapy dolutegravir women in gestation or use in women of childbearing age who may be in a state of pregnancy is contraindicated, or women with antiretroviral therapy dolutegravir remaining in a state of pregnancy and should make suspension and change it.

\section{Conclusion}

To conclude there is insufficient scientific evidence to associate the use of dolutegravir with the occurrence of adverse effects on the mother and her children.

\section{Author contributions}

Search, selection of articles was made by Lorena Paredes and Isabel Vargas, evaluation and extraction of information by Diana Cabrera, Isabel Vargas and analysis was conducted by Isabel Vargas and Lorena Paredes Eduardo Mahecha reviewed the proposed work and the revision of the article; Jorge Ramos was responsible for searching, selecting items, monitor the preparation and review of the proposal, analysis, drafting and revising the manuscript.

\section{Acknowledgments}

None.

\section{Funding}

None.

\section{Conflicts of interest}

The authors declare no conflicts of interest.

\section{References}

1. Katzung BG, Trevor AJ. Basic and clinical pharmacology. 2016:1-1141.

2. Van der Galien R, Ter Heine R, Greupink R, et al. Pharmacokinetics of HIV-integrase inhibitors during pregnancy: mechanisms, clinical implications and knowledge gaps. Pharmacokinet Clin. 2018;58:309323.

3. Coll O, Suy A, Hernandez S, Pisa S, et al. Prenatal diagnosis in human immunodeficiency virus-infected women: A new screening program for chromosomal anomalies. Am J Obstet Gynecol. 2006;194(1):192-198.

4. Chilean Ministry of Health. Acquired Immunodeficiency Syndrome HIV/ AIDS. Clinical guidelines SERIES MINSAL Part 2Rev Chilena infectol. 2010;27(4):359-373.

5. Antiretroviral pregnancy registry. INC Research; 2018.

6. TWG-HIV. The EMA reports of birth defects in children born to mothers with HIVtaking dolutegravir; working group on HIV treatments (TWGHIV), an entity declared of public interest NGO Development. 2018.

7. Zash R, Makhema J, Shapiro RL. Neural-tube defects treatment with dolutegravir from the time of conception. N Engl J Med. 2018;379:979981.

8. Zash R, Jacobson DL, Diseko M, et al. Comparative safety of dolutegravir-based or efavirenz-based antiretroviral treatment in Botswana started during pregnancy: an observational study. Glob Health Lancet. 2018;6(7):E804-E810.

9. Grayhack C, Sheth A, Kirby O, et al. Evaluating outcomes of motherinfants pairs using dolutegravir treatment for HIV During Pregnancy. AIDS. 2018;32(14):2017-2021.

10. Mounce ML, Pontiggia L, Adams JL. A single-center retrospective cohort analysis of maternal and infant outcomes in HIV-infected mothers treated with integrase inhibitors during pregnancy. Ther Infect Dis. 2017;6(4):531-544.

11. Mulligan N, Best BM, Wang J, et al. Dolutegravir pharmacokinetics in pregnant and postpartum women living with HIV. AIDS. 2018;32(6):729737. 
12. Bornhede R, Soeria AS, Westling K, et al. Dolutegravir in pregnancyeffects on HIV-positive women and Their infants. Eur J Clin Microbiol. 2018;37(3):495-500

13. Simons R, Ruiter A, Kulasegaram R. Dolutegravir use in 181 Patients, 54 women and nine pregnancies - a real life experience. HIV Med. 2016;17 (Suppl 1):14-71.

14. Fellay J, Boubaker K, Ledergerber B, et al. Prevalence of adverse events associated with potent antiretroviral treatment: swiss HIV cohort study. Lancet. 2001;358(9290):1322-1327.

15. Cunningham FG, Leveno KJ, Bloom SL, et al. Williams Obstetrics. 24th ed. 2015:899-914.

16. Gardner DG, Shoback D. Greenspan basic and clinical endocrinology. 9th ed. 2012.

17. Hoffman C, Welz T, Sabranski M, et al. Higher rates of neuropsychiatric adverse events leading to discontinuation dolutegravir in women and older patients. HIV Med. 2016;18(1):56-63.

18. Thorne C, Favarato G, Peters M, et al. Pregnancy and neonatal outcomes following prenatal exposure to dolutegravir. 9th International AIDS Society Conference on HIV Science; 2017.

19. There JW, Levin M, Sondheimer JM, et al. Pediatric diagnosis and treatment. 19 th ed. 2010.

20. Department of Health and Human Services of the United States. HIV medications and their side effects HIV/AIDS. infoSIDA; 2017.

21. Stellbrink HJ, Reynes J, Lazzarin A, et al. Dolutegravir in antiretroviralnaive Adults with HIV-1: 96-week results from a randomized doseranging study. AIDS. 2013;17;27(11):1771-1778.

22. Elzi L, S Erb, Furrer $\mathrm{H}$, et al. Adverse events of raltegravir and dolutegravir. AIDS. 2017;31(13):1853-1858. 